(C) 1979 IEEE. Personal use of this material is permitted. However, permission to reprint/republish this material for advertising or promotional purposes or for creating new collective works for resale or redistribution to servers

or lists, or to reuse any copyrighted component of this work in other works must be obtained from the IEEE.'

IEEE Transactions on Nuclear Science, Vol. NS-26, No. 3, June 1979

\title{
RECENT IMPROVEMENTS IN BEAM DIAGNOSTIC INSTRUMENTATION*
}

O. R. Sander, R. A. Jameson, and R. D. Patton

Los Alamos Scientific Laboratory, Los Alamos, NM 87545

\section{Abstract}

In high-current machines, such as LAMPF and the envisioned Hanford Engineering Development Laboratory (HEDL) Fusion Materials Irradiation Test (FMIT) Facility linac, hands-on maintenance is desired. Beam spill must be kept extremely low; therefore, attention must be given to beam fringes (tails). Beam matching to the structure becomes increasingly important. We describe equipment capable of accurately measuring transverse beam profiles over a range spanning more than four orders of magnitude and longitudinal phase profiles over ranges spanning more than three orders of magnitude. Errors in $100-\mathrm{MeV}$ transverse emittance measurements are explored and experimental emittance measurements made with three different methods are compared. Advantages of one nondestructive method are developed.

\section{High Resolution Transverse Profiles}

At LAMPF the operators typically tune the linac by steering the beam to give minimum spill in the spill monitors. The best tune is often one that does not have the bean centroids along the machine axis. Apparently steering keeps tails or small secondary beams from hitting the linac apertures. These undesirable tails often are $0.1 \%$ or less of the total beam. In principle, these tails can be observed in profile measurements made using a wire scanner, which passes a wire across the beam and amplifies the secondary electron current created by the beam striking the wire. The observation of these small tails was complicated because the LAMPF control and analog data acquisition system (ADS) uses a 12-bit computer. The resulting resolution of 1 count in 2048 is insufficient to observe beam tails with standard linear amplifiers.

To solve this problem we constructed a multigain amplifier with four ranges, each differing by a factor of 10. Special care was required in the basic design, grounding, and shielding of these amplifiers, for we required sensitivities less than a nanoampere in the presence of megawatt $\mathrm{rf}$ power systems. The circuit diagram and the grounding and shielding block diagrams are shown in Fig. 1. In a profile measurement the wire scanner drives a vertical and a horizontal wire

*Work supported by the U. S. Department of Energy. through the beam at $45^{\circ}$ to the vertical. The grounding and biasing module negatively biases the wire of interest and grounds the other wire. This biasing prevents secondary electrons from the grounded wire from being recollected by the negative wire. The electron recollection will distort the measured profiles. Data acquisition is completely computer controlled with the gains switched to optimize the ADS counts. A computerized output of a wire scan, showing a useful range in excess of four orders of magnitude, is demonstrated in Fig. 2. The multigain amplifier has the added convenience of being applicable over a wide range of peak beam currents.

\section{High Resolution Longitudinal Phase Profiles}

As higher peak and average currents are accelerated at LAMPF, the longitudinal tuning becomes more critical for maintaining minimal spill along the linac. Observance of undesirable tails in longitudinal space is required if they are to be eliminated. Because of the difficulty of directly measuring the longitudinal emittance, we measure only the longitudinal phase profile using the phase scan method. Beam tails should be observable in these profiles. The emittance could be reconstructed using the techniques outlined below.

In the LAMPF $805-\mathrm{MHz}$ linac, the phase scan method consists of sweeping the phase of the first two modules $(100-125 \mathrm{MeV})$ and of recording the amount of beam that is captured and accelerated by these modules. The captured beam has sufficient energy to transverse a copper absorber and stop in a copper collector downstream. The standard procedure is to measure the collector current with an amplifier whose range is matched to the peak beam current. As the left edge of the machine acceptance is swept across the beam, the collector current increases from zero (no beam within the acceptance) to full peak beam current (all beam inside the acceptance). This collector current is the integral of the captured beam. Differentiation of the current with respect to the phase yields the phase profile or the longitudinal emittance projected onto the phase axis. This method is limited also by the 12-bit ADS system. It is impossible to use either single or multigain amplifiers to obtain high resolution at the upper end of the integral, when most of the beam is on the collector.

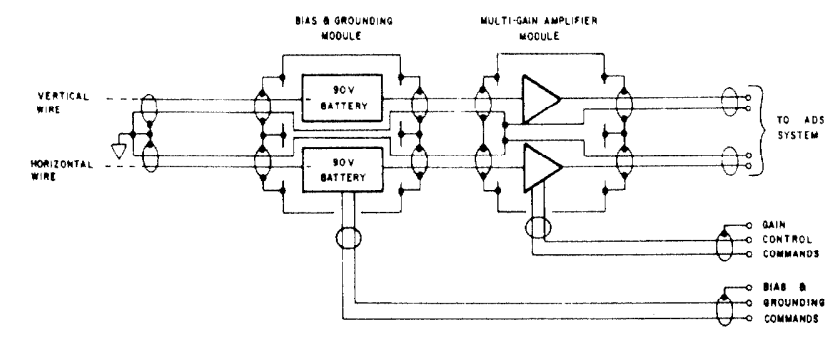

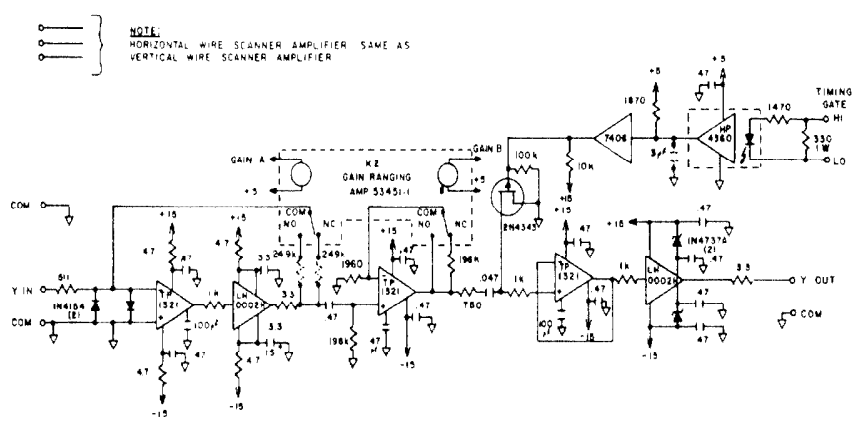

Fig. 1. Multigain amplifier circuit.

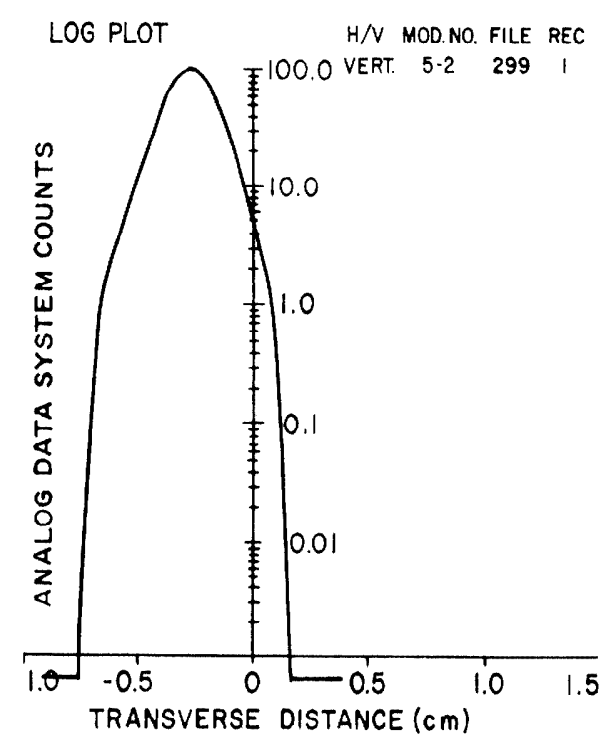

Fig. 2. Beam profile from raw data. 
Using a slide-back current amplifier we overcame this difficulty. A portion of the sum of the absorber and collector currents, i.e., the total peak current, is subtracted from the collector current. Using the total peak current eliminates the first-order effects of small peak-beam fluctuations. The circuit diagram of the slide-back amplifier is shown in Fig. 3. We minimize the current difference and measure it with our multigain amplifier. The improved sensitivity thus gained is a factor 250 over that of the standard method; the sensitivity is $0.01 \mu \mathrm{A}$ when the peak current is $5 \mathrm{~mA}$. With this approach we detect both leading and trailing tails of the phase profile. A phase profile with a resolution of better than 1 part in $10^{3}$ is shown in Fig. 4. The distribution has a degree of jaggedness that cannot be associated with beam fluctuations or statistics. This jaggedness is caused by different time responses in various portions of the electronics that cause offsets to occur when a time-dependent beam current is present. This problem has been corrected and the apparatus awaits further testing.

\section{Examination of Transverse Emittance Measuring Methods}

The ability to match the beam emittance to the linac acceptance is basic to understanding and operating a linac. Linac acceptances are typically gained from computer simulation models; bean emittances are gained from direct measurements. The usual method of emittance measurement consists of moving a slit across the beam and simultaneously observing the divergence of the transmitted portion of the beam on a collector that consists of parallel conducting strips, isolated from each other and mounted on a backing plate. Current caused by secondary emission of electrons from the strips is detected and assumed to be directly proportional to the amount of beam striking the strip. We examined this method by comparing its results with those of two different methods and have explored possible sources of error.

\section{Method}

The transverse emittance of the LAMPF beam at $100 \mathrm{MeV}$ was measured using (1) the usual slit and collector method, (2) the same slit and a wire scanner placed four times further downstream than the collector in method (1), and (3) an emittance reconstruction based on three profile measurements made at three locations separated by drift spaces. In method (3) a modified LASL RECON ${ }^{1}$ family of codes was used; these codes employ the multiplicative algebraic reconstruction technique, MART. We used data from the three methods to generate plots of total emittance and rms ellipse beam parameters versus the percentage of total

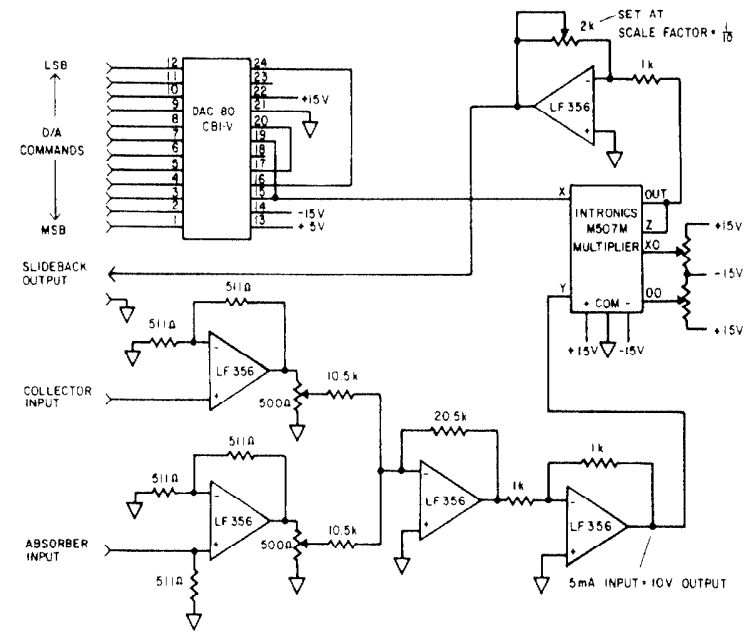

Fig. 3. Slide-back amplifier circuit diagram. beam. We used extrapolation techniques ${ }^{2}$ to determine the $100 \%$ value of the beam.

\section{Results and Discussion}

Methods (1) and (2) were compared by considering errors caused by angular resolution; ${ }^{3}$ finite slit size; ${ }^{4}$ number of samples; ${ }^{3}$ possible collector construction related effects, e.g., accumulation of charge on the backing plate insulator and communication between strips on the collector by the secondary emission electrons around the collector; and spacecharge effects ${ }^{5}$ on the transmitted strip of beam.

For method (1), the total emittance from raw data was consistently greater than that of method (2); see Fig. 5. All the above mentioned errors except those caused by the collector construction were examined and only the error caused by the finite slit size was sig nificant. The measured total emittance $E_{m}$ is given by $^{3}$

$E_{m}=E_{t}\left[1+\frac{2 b^{2}}{3 E_{t}}\left(\frac{\beta t}{2 L^{2}}+\frac{\gamma t}{2}-\frac{\alpha t}{L}\right)\right]^{1 / 2}$

where $E_{t}, \beta_{t}, \gamma_{t}, \alpha_{t}$ are the true total emittance and ellipse parameters in the C.S. notation, $b$ is the slit width $(0.0635 \mathrm{~cm})$, and $\mathrm{L}$ is the distance between the slit and the detector. Because of the large value of $\mathrm{L}(485 \mathrm{~cm})$ in method (2) the difference between $E_{m}$ and $E_{t}$ is negligible. With removal of this error in method (1) total emittance plots using all three methods were in excellent agreement. We conclude that the collector construction effects are negligible. In addition, the agreement between the first two methods and the third indicates that the neutralization of the beam by back-streaming secondary emission electrons from the slit is also negligible. Neutralization effects have been observed el sewhere 6 but with significantly higher intensity and lower energy $(750 \mathrm{keV})$ beams.

Methods (1) (corrected) and (3) were compared to method (2) by calculating $\Delta R / R, 4$ the relative residual betatron os cillation for each method; to compare the shape factors, $\alpha$ and $\beta$, see Fig. 6 . The true ellipse parameters $\alpha, \beta$ were assumed equal to those in method (2). For purposes of beam matching, a 0.1 value for $\Delta R / R$ is considered very good. The small but significant disagreement between the results of methods (2) and (3) is apparently caused by artifact production of the RECON code. This artificial distortion of the fitted ellipse may be removed when

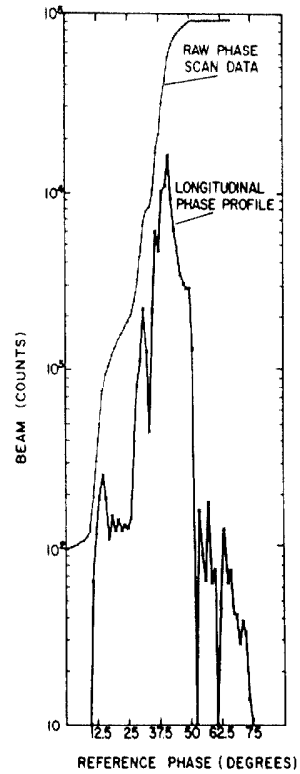

Fig. 4. Phase scan data and phase profile. 


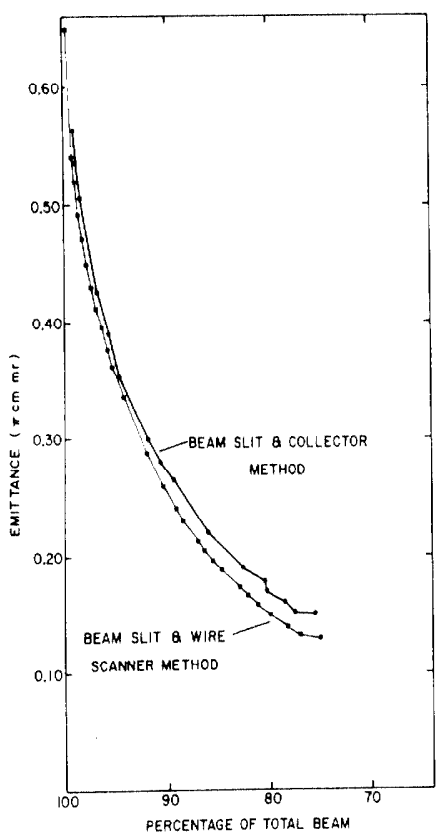

Fig. 5. Total emittance as a function or percentage of beam.

improved transformation methods ${ }^{7}$ are incorporated into $\mathrm{RECON}$.

\section{Future Emittance Measurements}

LAMPF tune-up procedures are typically done at 1to $3-\mathrm{Hz}$ repetition rates compared to a $120-\mathrm{Hz}$ production operation rate. This 1 imitation results from the use of destructive emittance measuring devices, the slit and collector type, which would not survive in full production heam. To measure emittance at full intensity and at $120-\mathrm{Hz}$ is desirable.

We found that wire scanners equipped with 1.3-mil carbon wire can give reliable beam profiles at $100 \mathrm{MeV}$ from beams in excess of 450- $\mu \mathrm{A}$ average current and can do so without causing unacceptable losses along the linac.

These tests and the apparent success of the reconstruction technique based on three profiles have encouraged us to use this technique to measure emittances of production beams with high average current. Although the accuracy of this method decreases when the emittance structure becomes complicated, experience has shown that beams with complicated structure have poor quality, give rise to losses along the linac, and should be corrected. Even under these conditions, this method would give valuable information. Production beam measurement al so requires reconstruction using the actual operating transfer functions between profile measurement stations, so the accuracy to which these functions are known will be important

We $\mathrm{plan}$ to use this method at $750 \mathrm{keV}$ and compare its results with those found using the standard destructive method. At $750 \mathrm{keV}$, space-charge effects and beam neutralization caused by the slit will be more important.

The reconstruction method also can be used for longitudinal emittance measurements, using the appropriate transfer functions between measurements. This method also is being considered for measuring the transverse emittance of the 100-mA cw linac being built for the HEDL FMIT project. ${ }^{8}$ Because the high average current of this linac precludes the use of wire scanners, the feasibility of obtaining profiles by detecting the light given off in the recombination

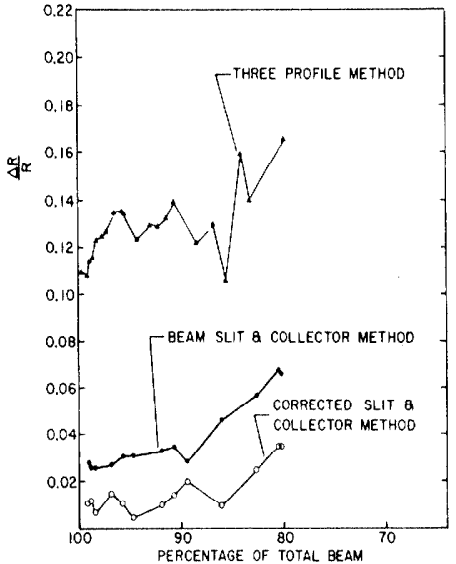

Fig. 6. Comparison of reconstructed ellipses.

of residual gases, ionized by the beam, is being investigated. Finally, we are examining the general RECON reconstruction method to reconstruct four- and six-dimensional phase-space distributions from combinations of two-dimensional measured distributions. We are seeking methods for assigning particle coordinates in computer simulation codes.

\section{Conclusion}

A method was presented for measuring transverse beam profiles ranging over four orders of magnitude. This method uses a multigain amplifier whose gain range is computer selected to optimally use the 12-bit analog data system. A method to measure longitudinal phase profiles over a range in excess of three orders of magnitude was also presented; it uses a slide-back amplifier and the multigain amplifier. Emittance measurements of a $100-\mathrm{MeV}$, 5-mA peak current beam were examined using standard slit and collector techniques and a reconstruction method based on three profiles. The only significant source of error was the finite slit size. The advantages of the reconstruction method were developed; the principal advantage was the ability to measure the emittance of beams with highaverage currents.

\section{References}

1. J. S. Fraser, "Beam Tomography or ART in Accelerator Physics," Los Alamos Scientific Laboratory report LA-7498-MS (Nov. 1978).

2. R. A. Jameson, editor, "Space Charge in Linear Accelerators Workshop," Los Alamos Scientific Laboratory report LA-7265-6 (May 1978).

3. J. Guyard and M. Weiss, "Use of Beam Emittance Measurements in Matching Problems," Proc. 19th Proton Linear Acc. Conf., Chalk River, Canada, AECA 5677, Nov. 1974.

4. R. Gluckstern, University of Maryland, private communication (May 1978).

5. J. Guyard and L. Marce, "Parametres Significatifs d'un Faisccau de Protons A $500 \mathrm{keV}$ Issus de la Mesure des Moyennes Quadratiques," CERN Report MPS/LIN/75-1, Juin 1975.

6. L. Evans and D. Warner, "A Critical Study of Emittance Measurements of Intense Low Energy Beams," NIM 104 (6l-70) 1972.

7. G. Minerbo, "MENT: A Maximum Entropy Algorithm for Reconstructing a Source from Projection Data," to be published in Computer Graphics and Image Processing.

8. R. A. Jameson, "High Intensity Deuteron Linear Accelerator (FMIT)," to be presented at the 1979 Particle Accelerator Conference, San Francisco, CA, March 12-14, 1979. 